\title{
RESEARCH NOTES
}

\section{SOME REMARKS ON THE STABILITY ANALYSIS IN ROBE'S THREE BODY PROBLEM}

\author{
R. MEIRE \\ Astronomical Observatory \\ Ghent State University \\ Krijgslaan 271 - S9 \\ $9000 \quad$ GENT \\ BELGIUM \\ (Received October 12, 1979)
}

ABSTRACT. An improved technique is presented for the stability analysis of Robe's 3-body problem which gives more accurate results for the transition curves in the parameter plane than does Robe's paper.

A novel property of the system of differential equations describing the motion is used, which reduces the computer time by more than $50 \%$.

KEY WORUS AND PHRASES. 3-body problem, stabilety, transetion curve, Floquet-theory. 1980 MATHEMATICS SUBJECT CLASSIFICATIUN CUDES. 85A05

\section{INTRODUCTION.}

In a recent paper Robe [1] presented a new kind of restricted three body problem, where one body $m_{1}$ is a rigid spherical shell, filled with an homogeneous incompressible fluid of density $\rho_{1}$, where a second body $m_{2}$ is a mass point outside the shell, and where $m_{3}$ is a small solid sphere of density $\rho_{3}$, restricted to move inside the shell, its motion determined by the attraction of $\mathrm{m}_{2}$ and the buoyancy force due to the fluid $\rho_{1}$.

There exists a solution with $\mathrm{m}_{3}$ at the center of the shell while $\mathrm{m}_{2}$ describes a Keplerian orbit around it. Robe investigated the stability of this configuration under the assumption that the mass of $\mathrm{m}_{3}$ is infinitesimal. The linearized equations 
of motion in the neighborhood of this equilibrium are

$$
\begin{aligned}
& \ddot{x}-2 \dot{y}=\left\{\frac{1+2 \mu}{1+e \cos v}-\frac{K\left(1-e^{2}\right)^{3}}{(1+e \cos v)^{4}}\right\} x \\
& \ddot{y}+2 \dot{x}=\left\{\frac{1-\mu}{1+e \cos v}-\frac{K\left(1-e^{2}\right)^{3}}{(1+e \cos v)^{4}} \quad y\right. \\
& \ddot{z}+z=\left\{\frac{1-\mu}{1+e \cos v}-\frac{K\left(1-e^{2}\right)^{3}}{(1+e \cos v)^{4}} z\right.
\end{aligned}
$$

where

$$
\begin{aligned}
& \mathrm{K}=\frac{4 \pi}{3} \cdot \frac{\rho_{1} \mathrm{a}^{3}}{\left(\mathrm{~m}_{1}+\mathrm{m}_{2}\right)}\left(1-\frac{\rho_{1}}{\rho_{3}}\right) \\
& \mu=\frac{\mathrm{m}_{2}}{\left(\mathrm{~m}_{1}^{*}+\mathrm{m}_{2}\right)} \\
& \mathrm{a}=\text { semi-major axis of the Keplerian orbit. }
\end{aligned}
$$

These equations are referred to a coordinate system $0 x y z$, where 0 is the center of $\mathrm{m}_{1}$, Ox points to $\mathrm{m}_{2}$ and $\mathrm{Oxy}$ is the plane of the Keplerian orbit.

$$
\text { If } \rho_{1}=0 \text { (shell empty) or } \rho_{1}=\rho_{3} \text {, then } k=0 \text { and the equations of motion }
$$
become

$$
\begin{aligned}
& \ddot{x}-2 \dot{y}=(1+2 \mu) r x \\
& \ddot{y}+2 \dot{x}=(1-\mu) r y \\
& \ddot{z}+z=(1-\mu) r z
\end{aligned}
$$

with $r=\frac{1}{1+e \cos v}$.

Equations (1.4) and (1.5) describe the motion in the orbital plane. Robe investigated the stability in the orbital plane by means of the Floquet-theory. However, one can separate the fourth-order system $(1.4),(1.5)$ into two independent second-order systems.

2. THE TRANSFORMATION TO SECOND-ORDER SYSTEMS.

$$
\text { Using } \quad \xi=\left[\begin{array}{l}
\mathrm{x} \\
\mathrm{y}
\end{array}\right] \text { and } n=\left[\begin{array}{c}
\dot{\mathrm{x}} \\
\dot{\mathrm{y}}
\end{array}\right] \text {, }
$$

equations (1.4) and (1.5) can be written 


$$
\left[\begin{array}{c}
\dot{\xi} \\
\dot{n}
\end{array}\right]=\left[\begin{array}{cc}
0 & \mathrm{E} \\
\mathrm{rC}_{0} & 2 \mathrm{D}
\end{array}\right]\left[\begin{array}{l}
\xi \\
n
\end{array}\right]
$$

where

$$
E=\left[\begin{array}{ll}
1 & 0 \\
0 & 1
\end{array}\right], \quad D=\left[\begin{array}{cc}
0 & 1 \\
-1 & 0
\end{array}\right]
$$

and

$$
C_{0}=\left[\begin{array}{cc}
1+2 \mu & 0 \\
0 & 1-\mu
\end{array}\right]
$$

Now we make the following transformation (Tschauner [2])

$$
\left[\begin{array}{l}
\xi \\
n
\end{array}\right]=\left[\begin{array}{cc}
\mathrm{E} & \mathrm{E} \\
\mathrm{P}_{1} & \mathrm{P}_{2}
\end{array}\right]\left[\begin{array}{l}
\delta \\
\varepsilon
\end{array}\right]
$$

and obtain

$$
\left[\begin{array}{c}
\dot{\delta} \\
\dot{\varepsilon}
\end{array}\right]=\frac{1}{\mathrm{P}_{2}-\mathrm{P}_{1}} \cdot\left[\begin{array}{cc}
\mathrm{P}_{2} \mathrm{P}_{1}-\mathrm{rC}_{0}-2 \mathrm{DP}_{1}+\mathrm{P}_{1}^{\prime} & \mathrm{P}_{2}^{2}-\mathrm{rC}_{0}-2 \mathrm{DP}_{2}+\mathrm{P}_{2}^{\prime} \\
-\mathrm{P}_{2}^{2}+\mathrm{rC}_{0}+2 \mathrm{DP}_{1}-\mathrm{P}_{1}^{\prime} & -\mathrm{P}_{1} \mathrm{P}_{2}+\mathrm{rC}_{0}+2 \mathrm{DP}_{2}-\mathrm{P}_{2}^{\prime}
\end{array}\right]\left[\begin{array}{c}
\delta \\
\varepsilon
\end{array}\right]
$$

Making the nondiagonal elements zero, we obtain

$$
\left[\begin{array}{c}
\dot{\delta} \\
\dot{\varepsilon}
\end{array}\right]=\left[\begin{array}{ll}
\mathrm{P}_{1} & \\
& \mathrm{P}_{2}
\end{array}\right]\left[\begin{array}{l}
\delta \\
\varepsilon
\end{array}\right]
$$

where $\mathrm{P}_{1}$ and $\mathrm{P}_{2}$ are two different solutions of the Riccati equation

$$
\dot{\mathrm{P}}=2 \mathrm{DP}-\mathrm{P}^{2}+\mathrm{rC}_{0} \text {. }
$$

Using

$$
P=\left[\begin{array}{ll}
\mathrm{p}_{11} & \mathrm{p}_{12} \\
\mathrm{p}_{21} & \mathrm{p}_{22}
\end{array}\right] \text {, }
$$


equation (2.4) becomes

$$
\begin{aligned}
& \dot{\mathrm{p}}_{11}=2 \mathrm{p}_{21}-\mathrm{p}_{11}^{2}-\mathrm{p}_{12} \mathrm{p}_{21}+\mathrm{r}(1+2 \mu) \\
& \dot{\mathrm{p}}_{22}=-2 \mathrm{p}_{12}-\mathrm{p}_{22}^{2}-\mathrm{p}_{12} \mathrm{p}_{21}+\mathrm{r}(1-\mu) \\
& \dot{\mathrm{p}}_{12}=2 \mathrm{p}_{22}-\mathrm{p}_{12}\left(\mathrm{p}_{11}+\mathrm{p}_{22}\right) \\
& \dot{\mathrm{p}}_{21}=-2 \mathrm{p}_{11}-\mathrm{p}_{21}\left(\mathrm{p}_{11}+\mathrm{p}_{22}\right)
\end{aligned}
$$

Now let

$$
\begin{aligned}
& \mathrm{p}_{11}=\mathrm{w}+\mathrm{z} \\
& \mathrm{p}_{22}=\mathrm{w}-\mathrm{z} \\
& \mathrm{p}_{12}=\mathrm{u}-\mathrm{v}+1 \\
& \mathrm{p}_{21}=\mathrm{u}+\mathrm{v}-1
\end{aligned}
$$

Then equation (2.5) becomes

$$
\begin{aligned}
& \dot{\mathrm{w}}=-1-\mathrm{w}^{2}-\mathrm{z}^{2}-\mathrm{u}^{2}+\mathrm{v}^{2}+\mathrm{r}\left(\frac{2+\mathrm{u}}{2}\right) \\
& \dot{\mathrm{z}}=2 \mathrm{u}-2 \mathrm{wz}+\mathrm{r} \frac{3}{2} \mathrm{u} \\
& \dot{\mathrm{u}}=-2 \mathrm{z}-2 \mathrm{uw} \\
& \dot{\mathrm{v}}=-2 \mathrm{vw}
\end{aligned}
$$

The last two equations yield

$$
\left.\begin{array}{l}
w=\frac{v}{2}\left(\frac{i}{v}\right) \\
z=-\frac{v}{2}\left(\frac{\dot{u}}{v}\right)
\end{array}\right\}
$$

If we use $p=\frac{1}{v}$ and $q=\frac{u}{v}$, we get

$$
\left.\begin{array}{l}
w=\frac{\dot{p}}{2 p} \\
z=-\frac{\dot{q}}{2 p}
\end{array}\right\}
$$


Substituting (2.8) into the first two equations of (2.7), we have

$$
\begin{aligned}
& p \cdot \ddot{p}-\frac{1}{2} \dot{p}^{2}+[L-r(2+\mu)] p^{2}-2=-2\left(q^{2}+\frac{1}{4} \dot{q}^{2}\right) \\
& \ddot{q}+4 q=-3 r p \mu
\end{aligned}
$$

Now if we let

$$
r p=k_{0}+k_{1} \cos v
$$

we find as a solution for (2.10)

$$
q=-\frac{3}{4} \mu k_{0}-\mu k_{1} \cos v+k_{2} \cos ^{2} v
$$

If we substitute the solutions (2.11) and (2.12) into (2.9), we obtain (by identification) the values for $k_{0}, k_{1}$ and $k_{2}$ as functions of $\mu$ and $e$.

For $\mathrm{k}_{0}$, we obtain

$$
\mathrm{k}_{0}= \pm \frac{4}{\mathrm{c}}
$$

$$
\text { with } c=\sqrt{\frac{4 e^{4}}{(3 \mu+1)^{2}}+\frac{4 e^{2} \mu}{(3 \mu+1)^{2}}-(\mu+3)+\mu(9 \mu-8)}
$$

This will yield two different solutions, $P_{1}$ and $P_{2}$, if $c \neq 0$ and $c^{2}>0$.

So $c(\mu, e)=0$ will give us (analytically) a transtion curve in the $\mu-c$ plane, which corresponds to one of the transition curves (IE) that Robe obtained numerica11y.

This curve can be written as

$$
\frac{4 e^{4}}{(3 \mu+1)^{2}}+\frac{4 e^{2} \mu}{(3 \mu+1)^{2}}(\mu+3)+\mu \cdot(9 \mu-8)=0
$$

The elements $\mathrm{P}_{i j}$ of $\mathrm{P}_{1}$ and $\mathrm{P}_{2}$ are

$$
\mathrm{p}_{11}=\frac{-(2 \mu+1) \mathrm{e} \text { sinv }}{(3 \mu+1+\text { ecosv })(1+\text { ecosv })}
$$




$$
\begin{aligned}
& \mathrm{p}_{22}=\frac{-(\mu+1+2 e \cos v) \text { esinv }}{(3 \mu+1 \text { ecosv })(1+\text { ecosv })} \\
& \mathrm{p}_{12}=\frac{(3 \mu+1)\left(1-\frac{3}{4} \mu \pm \frac{\mathrm{c}}{4}\right)+\frac{\mathrm{e}^{2}}{2}+2 \mathrm{e}(\mu+1) \cos \mathrm{v}+\mathrm{e}^{2} \cos 2 \mathrm{v}}{(3 \mu+1+\mathrm{ecos} v)(1+\mathrm{ecos} v)} \\
& \mathrm{p}_{21}=\frac{-\left[(3 \mu+1)\left(1+\frac{3}{4} \mu \pm \frac{\mathrm{c}}{4}\right)+\frac{\mathrm{e}^{2}}{2}+2(2 \mu+1) \operatorname{ecosv}\right]}{(3 \mu+1+\mathrm{ecos} v)(1+\text { ecosv })}
\end{aligned}
$$

\section{STABILITY ANALYSIS.}

Now we perform a stability analysis using the Floquet theory, on the two independent second order systems (2.3)

$$
\begin{aligned}
& \dot{\delta}=\mathrm{P}_{1} \delta \\
& \dot{\varepsilon}=\mathrm{P}_{2} \varepsilon
\end{aligned}
$$

Both equations admit solutions for which

$$
u(v+2 \pi)=s_{i} u(v) \quad(i=1,2)
$$

where $s_{i}$ are the roots of the characteristic equation

$$
\operatorname{det}\left[X^{-1}(v) \cdot X(v+2 \pi)-s E\right]=0
$$

where $X(v)$ is a fundamental solution matrix of (3.1) (or (3.2)). Equation (3.3) can be written

$$
s^{2}-2 \alpha s+1=0
$$

For stable solutions,

$$
|\alpha|<1
$$

Thus the transition curves in the ( $\mu-e)$ plane, separating stable and non-stable regions, will be given by

or

$$
\begin{aligned}
& |\alpha|=1 \\
& s= \pm 1
\end{aligned}
$$

Taking $\mathrm{X}(\mathrm{v}=0)=\mathrm{E}$, equation $(3.3)$ becomes

where $X(2 \pi)=\left[\begin{array}{ll}\alpha & \beta \\ \gamma & \alpha\end{array}\right]$ is the monodromy-matrix and $\alpha^{2}-\beta \gamma=1$.

It can be shown (R. Meire and A. Vanderbauwhede [3]) that 
where

$$
\begin{aligned}
& X(2 \pi)=\operatorname{SX}^{-1}(\pi) \operatorname{SX}(\pi) \\
& S=\left[\begin{array}{cc}
1 & 0 \\
0 & -1
\end{array}\right]
\end{aligned}
$$

This implies that we only have to integrate the equations over $\pi$ instead of $2 \pi$. One can also prove that for $s= \pm 1$, one of the elements of $X(\pi)$ becomes zero which saves an additional $10 \%$ of computer time.

4. RESULTS.

We applied this method to the equations of (3.1) and (3.2). Equation (3.1) yielded two transition curves: FM and FE. Equation (3.2) yielded one transition curve HM. All results are given in Fig. 1.

The curve IE is the analytically obtained curve from equation (2.14). Along

$$
\left\{\begin{array}{llll}
\text { FM } & \beta=0 & \text { and } & \alpha=-1 \\
\text { FE } \gamma=0 & \text { and } & \alpha=-1 \\
\text { HM } \beta=\gamma=0 & \text { and } & \alpha=1
\end{array}\right.
$$

The intersection of the curves FE and IE can be obtained very accurately (Robe was not able to give precise coordinates).

The point $\mathrm{E}$ is determined as that point on the curve IE for which the characteristic roots of $(3.1)$ are -1 . The coordinates of the interesting points in the $\mu-e$ plane are

$$
\begin{aligned}
& \text { point } F\left\{\begin{array}{l}
\mu=0.928053 \ldots \ldots=\frac{5+\sqrt{97}}{16} \\
e=0
\end{array}\right. \\
& \text { point I }\left\{\begin{array}{l}
\mu=0.8888 \ldots=\frac{8}{9} \\
e=0
\end{array}\right. \\
& \text { point } E\left\{\begin{array}{l}
\mu=0.8596848 \\
e=0.4531741
\end{array}\right.
\end{aligned}
$$

The stable region consists of the shaded area in Fig. 1 and is now determined much 
more accurately than in Robe's paper where the fourth-order system (2.1) was used.

Fig. 1 : Stability-regions in the $\mu-e-p l a n e$

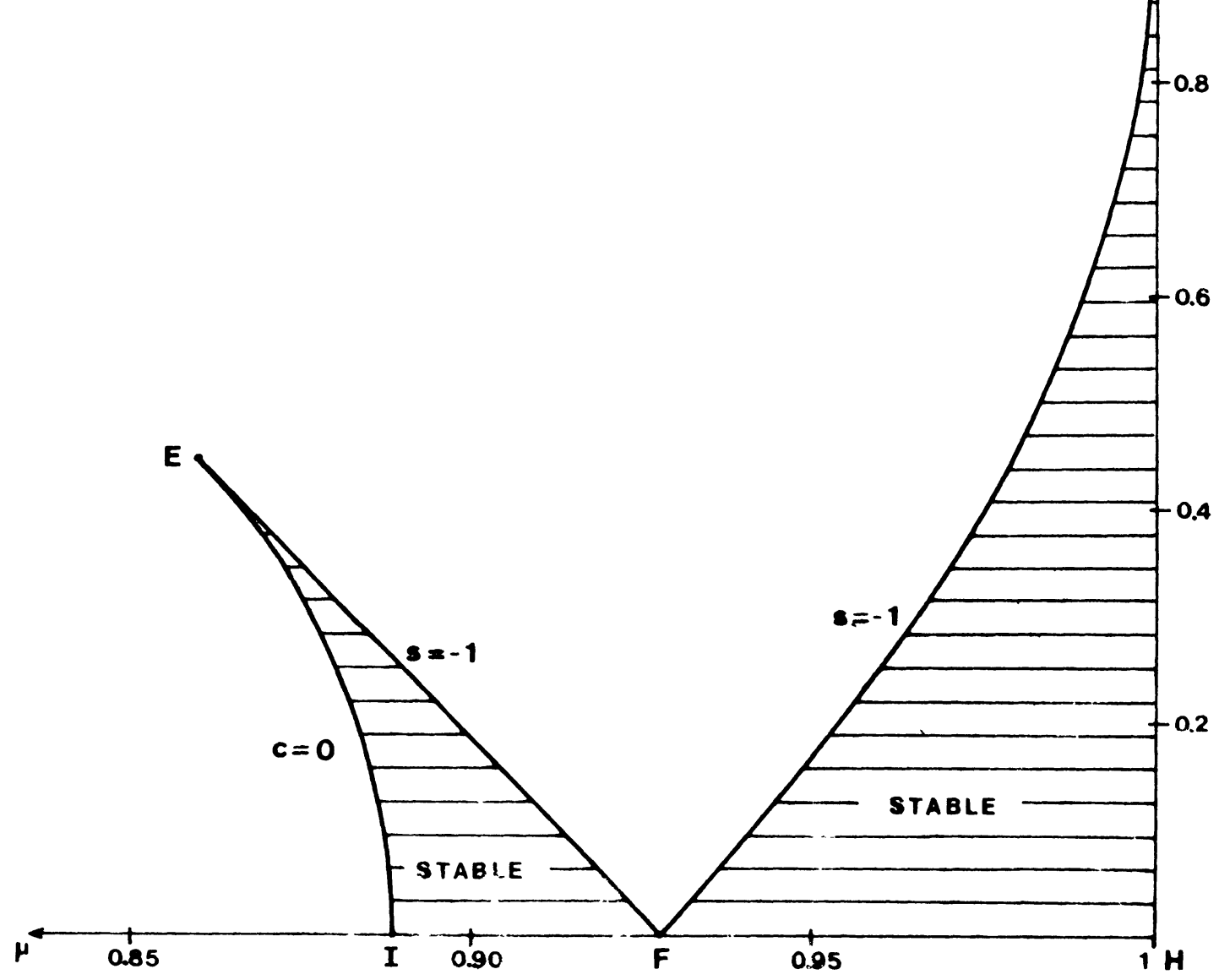

ACKNOWLEDGEMENTS.

The author thanks Prof. Dr. P. Dingens and Prof. Dr. H. Steyaert for reading the manuscript and for their valuable suggestions on this work.

\section{REFERENCES}

1. ROBE, H. A new kind of 3-body Problem, Ce1. Mech. 16 (1978), pp. 343-351.

2. TSCHAUNER, K. Die Aufspaltung der Variations-gleichungen des Elliptischen Eingeschränkten Dreikörperproblems, Ce1. Mech. 3 (1971), pp. 395-402.

3. MEIRE, R. and VANDERBAUWHEDE, A. A useful Result for Certain Linear Periodic Ordinary Differential Equations, J. Comp. and Applied Math. $\underline{5}, \mathrm{~N}^{\circ} 1$ (1979), pp. 59-61. 


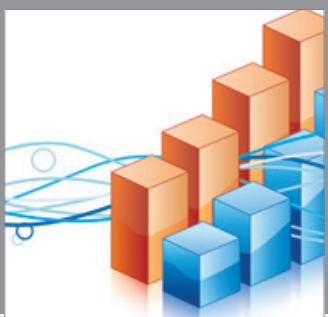

Advances in

Operations Research

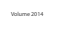

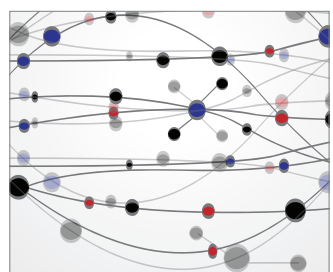

\section{The Scientific} World Journal
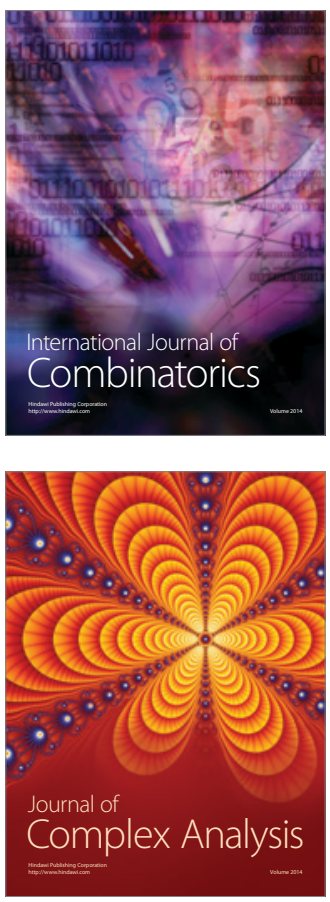

International Journal of

Mathematics and

Mathematical

Sciences
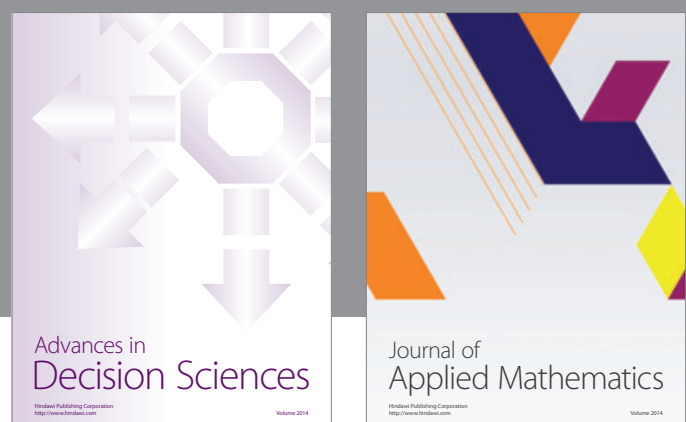

Journal of

Applied Mathematics
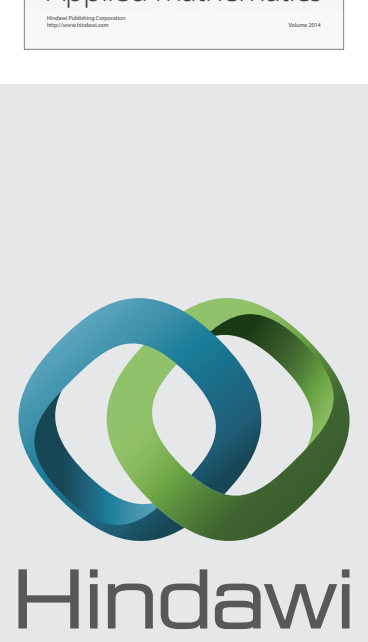

Submit your manuscripts at http://www.hindawi.com
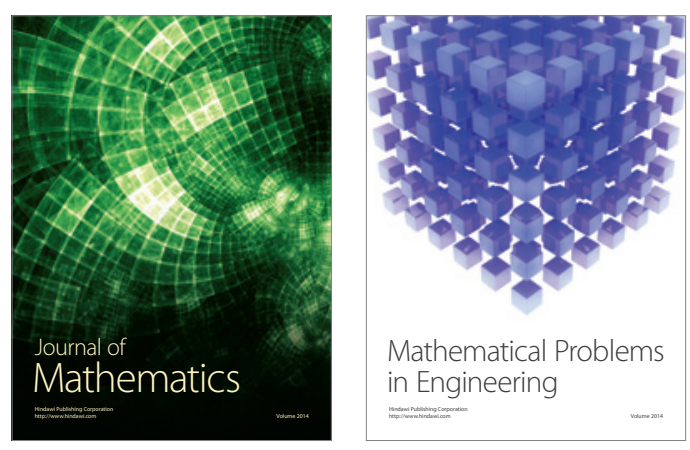

Mathematical Problems in Engineering
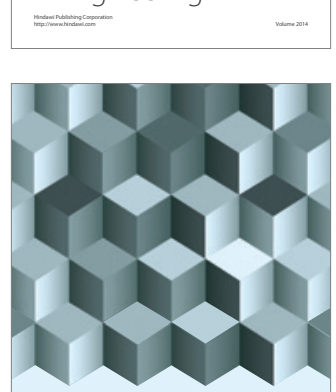

Journal of

Function Spaces
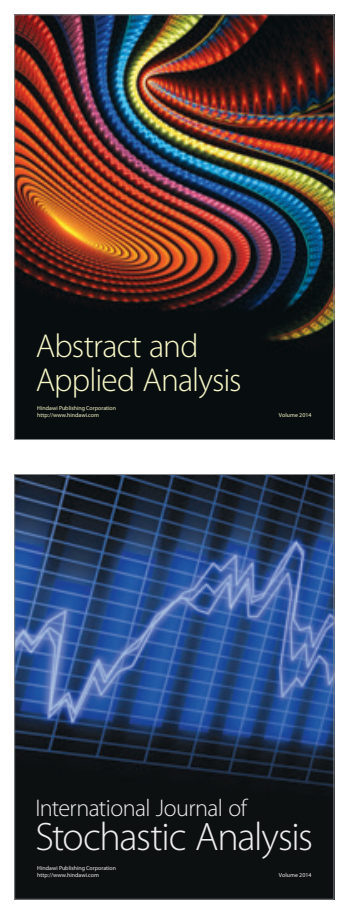

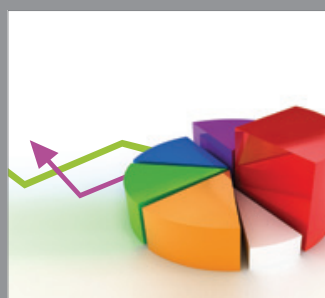

ournal of

Probability and Statistics

Promensencen
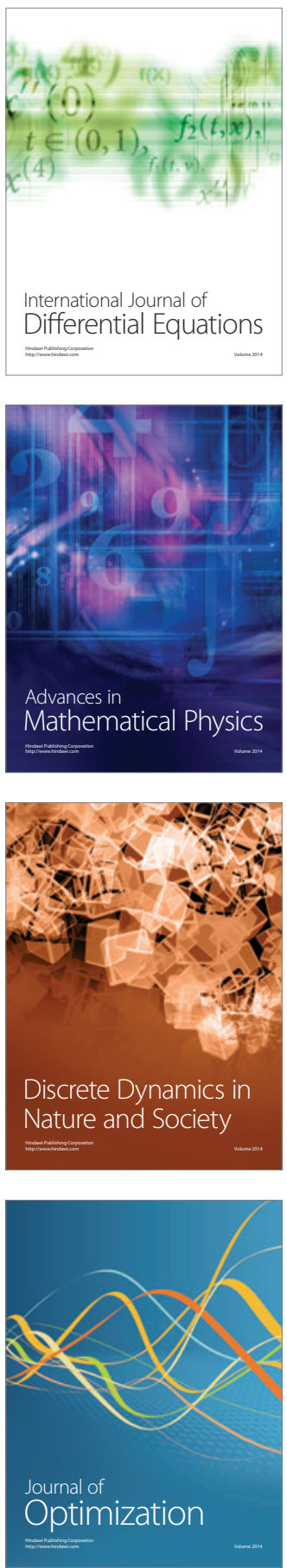